Bich, L. and Bechtel, W. (2022). Control Mechanisms: Explaining the Integration and Versatility of Biological Organisms. Adaptive Behavior. DOI: 10.1177/10597123221074429. Available at: https://journals.sagepub.com/doi/10.1177/10597123221074429

\title{
Control Mechanisms: \\ Explaining the Integration and Versatility of Biological Organisms
}

Leonardo Bich

ORCID: 0000-0002-2416-112X

IAS-Research Centre for Life, Mind and Society. Department of Philosophy. University of the Basque Country (UPV/EHU), Avenida de Tolosa 70, Donostia-San Sebastian, 20018, Spain.

*Corresponding author: leonardo.bich@ehu.es

William Bechtel

ORCID: 0000-0001-6370-5321

Department of Philosophy, University of California San Diego, La Jolla, CA, USA 92093

\begin{abstract}
:
Living organisms act as integrated wholes to maintain themselves. Individual actions can each be explained by characterizing the mechanisms that perform the activity. But these alone do not explain how various activities are coordinated and performed versatilely. We argue that this depends on a specific type of mechanism, a control mechanism. We develop an account of control by examining several extensively studied control mechanisms operative in the bacterium E. coli. On our analysis, what distinguishes a control mechanism from other mechanisms is that it relies on measuring one or more variables, which results in setting constraints in the control mechanism that determine its action on flexible constraints in other mechanisms. In the most basic arrangement, the measurement process directly determines the action of the control mechanism, but in more complex arrangements signals mediate between measurements and effectors. This opens the possibility of multiple responses to the same measurement and responses based on multiple measurements. It also allows crosstalk, resulting in networks of control mechanisms. Such networks integrate the behaviors of the organism but also present a challenge in tailoring responses to particular measurements. We discuss how integrated activity can still result in differential, versatile, responses.
\end{abstract}

Keywords: adaptive and versatile behavior; control mechanisms; crosstalk; integration; Escherichia coli.

\section{Introduction}

Organisms, from single-celled bacteria and archaea to multicellular plants and animals, are highly integrated and often respond in versatile and adaptive ways to circumstances they 
confront. As autonomous systems, they perform activities that suffice to construct, repair, and maintain themselves as cohesive entities far from equilibrium. Individual activities can be explained by identifying and characterizing the responsible mechanism. But that doesn't explain how they act as wholes in versatile and adaptive ways. That, we argue, results from a distinct kind of mechanism, a control mechanism. Control, however, is often conceptualized in relatively simple models, such as negative feedback. So conceived, control is insufficient to account for the versatility of organisms. In this paper we will draw upon examples of control processes in a relatively simple but extensively studied organism, the Gram-negative bacterium E. coli, to develop an account of the features of control mechanisms that enable organisms to achieve a high degree of integration while acting flexibly and adaptively.

Understanding control requires distinguishing between two types of mechanisms: control and controlled mechanisms. Controlled mechanisms are usually those production mechanisms involved in the basic activities such as the synthesis and repair of parts and the generation of energy. Control mechanisms operate on controlled mechanisms by modifying their activation state or modulating their activities. Control mechanisms can be controlled, in turn, by other control mechanisms. We identify four features of control mechanisms. The first is that it measures relevant variable conditions and uses the products of measurement to alter the functioning of mechanisms in the organism. A second characteristic is that a control mechanism performs these activities in a manner that is decoupled from the ongoing physiological processes it is controlling. A third feature is the inclusion of signals as intermediaries between the sensor and effector components of a control mechanism. These signals enable control mechanisms to respond to multiple sensors and to initiate multiple responses. When signals are shared between different control mechanisms, they enable organisms to coordinate complex responses to the varying array of conditions the organism encounters. Such an interconnected network of signals, however, presents its own problem - how to select between responses so as to effectively respond to specific conditions. This requires a fourth ingredient: imposing dynamic segregation on control components so that an appropriate set of responses can be realized.

While our account is motivated by focusing on what is regarded as a relatively simple organism, we argue that the features we identify can explain more generally the effectiveness of control in achieving integration and versatile, adaptive behavior in all organisms. Multicellular organisms rely on additional means of realizing control. In animals, neurons play a central role. They, however, realize networks with the same features as control mechanisms in $E$. coli. Thus, we propose that these features-(1) measuring appropriate variables and acting upon those measures in a manner that (2) is dynamically decoupled from basic metabolism, while (3) allowing for intermediate components to integrate different measurements and effects, and yet (4) enabling sufficient segregation to achieve specific responses-explains much of the integrated, versatile, adaptive behavior exhibited by biological organisms. 
Control, on our account, plays a central role in making living organisms integrated and versatile entities. However, it has not received much philosophical attention. ${ }^{1}$ In previous work we have argued that control provides a constructive bridge between two philosophical traditions in philosophy of biology-new mechanists and autonomy approaches (Bich \& Bechtel, 2021). The new mechanists have characterized mechanistic explanations that biologists in fields such as cell and molecular biology frequently advance to explain phenomena such as protein synthesis or production of ATP (Machamer, Darden, \& Craver, 2000; Bechtel \& Abrahamsen, 2005; Glennan, 2017) and have analyzed the strategies biologists employ in advancing such explanations (Bechtel \& Richardson, 1993/2010; Craver \& Darden, 2013). On these accounts, the project of explanation begins with the characterization of the phenomena, identifies a responsible mechanism, and looks within it to explain the phenomenon. It thus gives little consideration to how the phenomena to be explained relate to the life of the organism in which they occur. The autonomy tradition, represented by Maturana and Varela (1980) and Moreno and Mossio (2015), among others, focuses on organisms as entities that carry out the activities required to maintain themselves far from equilibrium - synthesizing and repairing all of their own components from matter and energy extracted from the environment. While emphasizing the theoretical demands on living organisms, the autonomy tradition has, for the most part, not addressed how the organism carries out the activities required of an autonomous system. One way to provide such an account would be to identify the responsible mechanisms, but historically autonomy theorists have resisted invoking mechanisms since they seem to be too rigid to account for the flexibility and versatility organisms must exhibit to maintain autonomy.

We have proposed that a focus on control provides a basis for understanding how mechanisms can be harnessed to enable organisms to be autonomous systems. A key step towards constructing such an account was taken by Winning and Bechtel (2018), who drew upon Pattee $(1973 / 2012)$ to characterize the entities and activities highlighted in new mechanist accounts as constraints on flows of free energy (see also Militello \& Moreno, 2018). Drawing upon the contributions of Hooker (2013; see also Kauffman, 2000) they developed an account in which constraints (e.g., a pipe in which water flows or a skeleton to which muscles are attached) enable outcomes that would not otherwise be obtained. They provided the basis for an account of control by differentiating fixed and flexible constraints. A pipe or a single bone is a fixed constraint. If all constraints are fixed, a given mechanism will perform its characteristic activity whenever resources, especially energy, are available. Flexible constraints (e.g., valves or joints) are ones that can take on different arrangements, thereby changing the work the mechanism performs. For example, flexible constraints can be arranged so that no free energy flows through the mechanism and no work is done. This creates the possibility of control. Drawing again on Pattee, Winning and Bechtel take a second step-characterizing a type of mechanism that exerts control over another mechanism. The control mechanism carries out its work on the constraints of the other mechanism as a result of measurements it takes of conditions either of the system in which the mechanism is contained or its environment. A third step is to understand control mechanisms in the context of biological autonomy: the activities that

\footnotetext{
${ }^{1}$ Few recent exceptions include Bich, Mossio, Ruiz-Mirazo, and Moreno (2016); Winning and Bechtel (2018); Ross (in press).
} 
control mechanisms perform on the basis of measurements contribute to the maintenance of the organisms that produces and maintains those very mechanisms (Bich et al., 2016).

In section 2 we discuss $E$. coli chemotaxis to illustrate this basic conception of a control mechanism as involving both a sensor capable of making measurements and an effector able to operate on flexible constraints of other mechanisms. Although chemotaxis has often been invoked in philosophical discussions, little attention has been paid to the complexity of the measurement processes employed. Given the multiplicity of measurement devices and the way they are modulated by the outcome of a given measurement, chemotactic behavior is more variable and adaptive than often portrayed. $E$. coli chemotaxis also illustrates a further feature of control that has been emphasized by Bich et al. (2016) - while the control mechanism must integrate with the metabolic processes of the organism, it must also be dynamically decoupled from it. A control mechanism is not just a mechanism that can be connected to another mechanism to produce a phenomenon. The operation of the control mechanism is determined by the measured conditions and it acts to alter the operation of other mechanisms. As we discuss in section 3 , this entails that the components used in control be set apart from those employed in metabolic processes. Otherwise, the control mechanism would be fully constrained by the dynamics of metabolism and not free to respond to the measured quantities. We illustrate how this decoupling is achieved with a second example in which multiple control processes acquire a measurement from an ongoing metabolic process and then carry out control activities independently from it.

In section 4 we turn to another feature illustrated by other control mechanisms in $E$. coli: while in the simplest cases of control, the sensor and effector are combined in a single molecule, with conformation changes induced in the molecule by the sensor acting to alter the effector component, intermediates can be employed to carry signals between sensors and effectors. This can greatly enhance the versatility and adaptiveness of the bacterium's behavior by further developing two features already introduced: a response can be generated from multiple measurements and give rise to a coordinated set of activities. In some cases each control mechanism employs distinct intermediates and so operates independently of the others. But in many cases, they share intermediates, sometimes giving rise to interconnected networks of control mechanisms. While such integration enhances the cohesiveness of the organism and the capacity for adaptive responses, it can prevent the organism from making specific responses when required. In section 5 we examine some of the ways in which $E$. coli maintains selectivity as well as integration, thereby enhancing the versatility achieved by control mechanisms.

In section 6 we draw together the features we have identified into a generalizable conception of a control mechanism that allows for versatile and adaptive behavior. Turning briefly to the animal nervous system, we show that it too is grounded in sensors and effectors and is decoupled from the physiological and behavioral mechanisms it controls. We also describe how, as a result of relying on volume transmitters and neuropeptides, it realizes a highly integrated network. Yet, various strategies are deployed to achieve sufficient segregation. We argue that the tradeoff between integration and segregation is a generic feature of biological 
control systems; the evolution of control mechanisms reflects a dynamic process of both enhancing integration and maintaining sufficient segregation. In section 6 we also defend our characterization of control processes as involving control mechanisms. As we note throughout, control mechanisms differ from the mechanisms they control in relying on measurements of variables and acting to alter the activity of other mechanisms. Moreover, while mechanisms are often viewed as discrete, control mechanisms are often highly integrated. Other concepts, such as pathway and network, are often invoked in discussing control processes. While acknowledging the usefulness of these other concepts, we argue that our account of control mechanisms provides the appropriate framework for understanding control ${ }^{2}$.

\section{Basic elements of control mechanisms: sensors and effectors}

We begin with the two essential elements of a control mechanism: (1) a sensor which sets the value of a constraint in the control mechanism as a result of measuring a variable, and (2) an effector that acts on a flexible constraint in another mechanism, altering how it operates. All mechanisms result in the performance of work through the constraint on the flow of free energy (Winning and Bechtel, 2018). What distinguishes a control mechanism is that the constraints that determine the work that is performed are determined by the measurement process and that the work that is performed results in changes in the constraints operative in other mechanisms. Examples of sensors are molecules whose conformation is altered as a result of binding to other molecules whose presence or concentration is being measured. Examples of effectors include transcription factors, which bind to DNA sequences and activate or inhibit the transcription of individual genes or operons, and kinases, which phosphorylate proteins (including themselves in the case of autokinases) thereby altering their conformation and hence their ability to catalyze reactions.

In principle, any coupling between measurement and action on the constraints of a controlled mechanism is possible. However, among the possible couplings are those in which the altered functioning of the controlled mechanism is what is required for the organism to maintain itself. Moreover, processes such as natural selection and learning will tend to favor such couplings. The presence of such coupling is what enables mechanisms to promote autonomy. The sensor measures variables whose values correspond to the need for specific activities to be performed

\footnotetext{
2 The notion of control has a long and diversified history, rooted in cybernetics and systems theory. Historically, one common way to look at control is in terms of the capability to modify the dynamics of a system toward a certain state. (Rosen, 1970). Another, proposed by Powers (1990; Marken \& Powers, 1989) is the capability to act on the effects of a disturbance so that a given variable is maintained stable and error is minimized. We share with Rosen the general view of control as a source of change, although we develop it within the context of an organism which must be maintained viable. We share with Powers the importance of looking at control from the point of view of the inner organization of the system and the importance of sensing the state of the system or the effects of disturbances of the system. However, we do not share his cybernetic focus on controlling variables, the view of control as a correction system, and the consequent equivalences between what is sensed and what is controlled, and between control and feedback (see Bich et al., 2016 for a discussion of the relationship between regulatory control and feedback). On our account, what is controlled are not variables, but the operation of production and control mechanisms. Moreover, our focus is on the integration and coordination of control mechanisms and on the variety of activities they can generate.
} 
and the resulting state of the sensor sets constraints that allow the control mechanism to alter the controlled mechanism to perform those activities.

We illustrate the sensor and effector processes of control mechanisms with E. coli chemotaxis, the control process that causes the bacterium to move towards nutrients while avoiding toxins. While this example has been much discussed, ${ }^{3}$ most accounts fail to discuss the complexity of the sensor processes that enable the bacterium to respond to move up or down gradients of nutrients and toxins and respond to multiple nutrients and toxins. We begin, though, with the effector component of $E$. coli control mechanism. The effector acts on the 5-10 flagella $E$. coli employ to move through their environment. Each flagellum is a complex mechanism that draws energy from the ion gradient across the cell membrane and constrains this energy to rotate the flagellum. Flagella can either rotate clockwise or counterclockwise depending on the particular configuration of constraints in it; when all flagella rotate counterclockwise, the flagella form a coherent structure that propels the bacterium forward. When some rotate clockwise, the flagella separate and provide no propulsion. Instead, the bacterium tumbles until all the flagella rotate counterclockwise again, propelling the bacteria forward in a new direction. FliM (Flagellar motor switch) proteins at the base of each motor are the relevant flexible constraints - when the effector protein CheY is phosphorylated, it binds to FliM, constraining the motor to rotate clockwise. When there is no phosphorylated CheY to bind, the constraints change and the motor rotates counterclockwise (Dyer, Vartanian, Zhou, \& Dahlquist, 2009).

The phosphorylation state of the effector protein CheY is determined by sensor processes. In $E$. coli these are five types of transmembrane methyl-accepting chemotaxis proteins (MCPs) located at one pole (Sourjik \& Wingreen, 2012; Bi \& Sourjik, 2018). As the name suggests, these proteins extend through the inner of E. coli's two membranes. Each type of MCP is capable of binding to a specific set of ligands. When ligands bind to the receptors, they induce a change in conformation which is transmitted down the length of the protein where, at the end, the MCP phosphorylates the protein CheA. The receptor complexes are organized into arrays consisting of six receptors (organized as pairs of trimers that may respond to the same or different ligands) that converge on one CheA and jointly determine its phosphorylation state. The complex of receptors together determines how much CheA is phosphorylated. CheA is itself a histidine kinase which phosphates $\mathrm{CheY}$, which as we discussed above, is the effector that determines the direction the motor rotates.

\footnotetext{
${ }^{3}$ The role of regulatory control of bacterial chemotaxis has been discussed, for example, in relation to minimal cognition (van Duijn, Keijzer, \& Franken, 2006; Bich \& Moreno, 2016; Bechtel \& Bich, 2021). For a comprehensive review on bacterial cognition see (Lyon, 2015).
} 


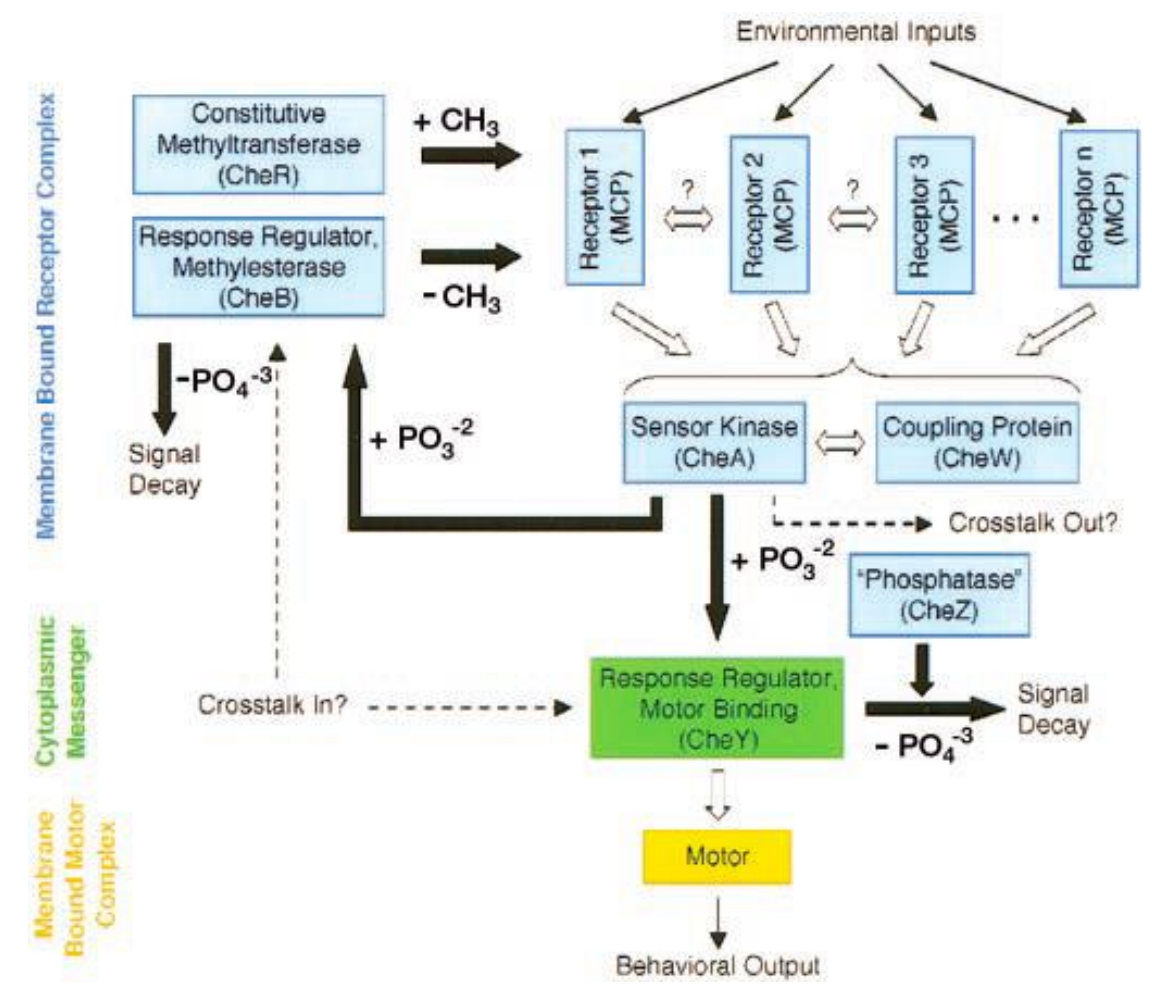

Figure 1. Abstract representation of the organization of the sensor complex of E. coli, showing the interaction between MCPs and the proteins CheA, CheW, Che R, CheB (upper part), the activation of the effector protein $\mathrm{CheY}$ in the cytoplasm (middle part) and its effect on the motor complex (lower part). Potential crosstalk with other control mechanisms is represented by dashed lines. From (Bourret \& Stock, 2002). Reproduced under Creative Commons License (CC BY-SA 4.0).

The combination of five sensors, each capable of measuring different chemicals as they bind to them, enables $E$. coli to integrate measurements of multiple nutrients and toxins in determining their responses (Figure 1). Individual bacteria vary in the numbers of each type of receptor they have; the result is that the sensors in different bacteria produce different output to the motor, resulting in different behavior in different bacteria. In addition, the various receptor arrays also interact in a manner that is not well understood.

The basic sensory mechanism described so far would enable $E$. coli to respond to the composite concentrations of various compounds in its environment, but it would not provide the bacterium with information about whether the concentrations of the individual ligands are increasing or decreasing. This is the information which the bacterium needs to determine whether it is approaching a nutrient or moving away from a toxin. To detect the gradient, E. coli employs two other proteins, CheR and CheB, to adapt each MCP to the current concentration of the ligand to which it responds. CheR constitutively adds methyl groups to each receptor, changing the receptor's conformation to render it more responsive over time. Recall that CheA is a histidine kinase-in addition to phosphorylating CheY, it also phosphorylates CheB. Phosphorylated CheB removes methyl groups from the receptors, countering the conformation change generated by CheR and making the MCPs less responsive. The overall effect is that the 
conformation of the MCPs changes in response to whether the bacterium is moving up or down the various gradients. This process of altering the conformation of the MCPs occurs on a slower timescale than that at which each processes new inputs and is extremely effective in allowing the bacterium to modulate its sensor so as to detect changes from the recent past. Webre, Wolanin, and Stock (2003) showed that the resulting sensor generates the same response to specific percentage of increase or decrease in ligands as the ligands vary over five orders of magnitude. ${ }^{4}$

Chemotactic control of the flagellum in E. coli illustrates the two basic requirements of a control mechanism-possession of a sensor process that performs measurements by altering its internal constraints in response to sensing the need for change, and of an effector process that, as a result of those constraints, produces changes on another mechanism. In carrying out these activities, control mechanisms must interact with those they control. But in order to act as controllers, they must, as we develop in the next section, be appropriately decoupled from those mechanisms.

\section{Control mechanisms rely on but are decoupled from the mechanisms they control}

Control processes are material processes. As such, they involve physical components that must be synthesized by the organism utilizing materials and free energy that the organism procures. Moreover, like all mechanisms, they constrain the flow of free energy so as to perform work. In making measurements, the control mechanism is causally responsive to the value of the variable being measured. And the work it performs is on the constraints of other mechanisms. In these respects, control mechanisms are interconnected with the ongoing metabolic and physiological activities of the organism and rely on them for their production and maintenance. Yet, to exercise control over these processes, there must also be a respect in which control processes are decoupled from these metabolic processes (Bich et al., 2016). Without decoupling, control mechanisms would simply be part of the productive activities constituting the organism.

What decoupling requires is that the effector processes execute actions based on the measurements made by the sensor processes. The constraints operative in the effector process are the result of the measurement. If the effectors' activities were themselves being determined by the ongoing metabolic activities of the organism, they would not be responding to what the sensor is measuring. Only to the extent that the constraints in the effector are the result of the measurement process is the effector acting as part of a control mechanism. ${ }^{5}$ Decoupling involves a kind of independence of the control mechanism from the mechanisms responsible for building, repairing, and replicating the organism. Control mechanisms do not contribute directly to any of these activities but only by altering the mechanisms that perform

\footnotetext{
${ }^{4}$ Bacteria thus conform to a relationship first discovered by Weber in human perception which was named Weber's law when it was represented mathematically by his student (Fechner, 1860).

${ }^{5}$ For simplicity, we treat decoupling as binary. It is possible for the effector of a control mechanism to be affected by both ongoing metabolism and the measurement made by the sensor. In such a case, only the constraint change in the effector brought about by the measurement would figure in the control process.
} 
the activities. They often constitute a physically distinct component of the organism, but even when not physically distinguishable, control mechanisms are conceptually differentiated in virtue of the measurement producing a state that determines how the effector alters the constraints in other mechanisms.

To illustrate how the sensor process of a control process can both make a measurement of ongoing metabolic processes and, based on that measurement, act through its effector process to alter constraints in mechanisms involved in physiological processes, we describe several control mechanisms that make measurements of states of components in the phosphoenolpyruvate-dependent phosphotransferase systems (PTSs) and through their effectors alter other metabolic mechanisms. These examples all illustrate decoupling in that the work performed by these mechanisms is determined by the measurements they make, which result in actions on flexible constraints in other mechanisms. In each case, the control mechanism is a step removed from ongoing metabolic activities.

PTSs provide a mechanism for incorporating a new molecule of sugar into the cell for one of the two molecules of phosphoenolpyruvate (PEP) that is metabolized in glycolysis. There is a separate PTS for each type of sugar molecule E. coli can use for energy. Each transfers a phosphate from the glycolytic intermediate PEP to a molecule of that type of sugar as it is being transported across the cell membrane and taken up into the glycolytic pathway (Figure 2 ). The key components of the transfer system are the proteins El and HPr and the protein complex Ell. Ell is different for each type of sugar; to date 21 different Ell complexes have been found in $E$. coli. PEP serves as both the energy source and the donor of the phosphate in this mechanism. El is a histidine autokinase that transfers the phosphate from PEP to one of its histidine residues (His 189). HPr, a histidine-containing phosphocarrier protein, then receives the phosphate from El and passes it to EIIA, which passes it to EIIB. ElIB then phosphorylates a sugar molecule as it crosses the membrane, preventing the sugar from passing back out of the cell. Each component rapidly transfers the phosphate to the next component in the pathway, with the result that when the cell is using a particular type of sugar, the intermediates for its PTS are most often in the unphosphorylated state. If, however, there is no sugar available to receive the phosphate, each remains in a phosphorylated state (Deutscher, Francke, \& Postma, 2006). 


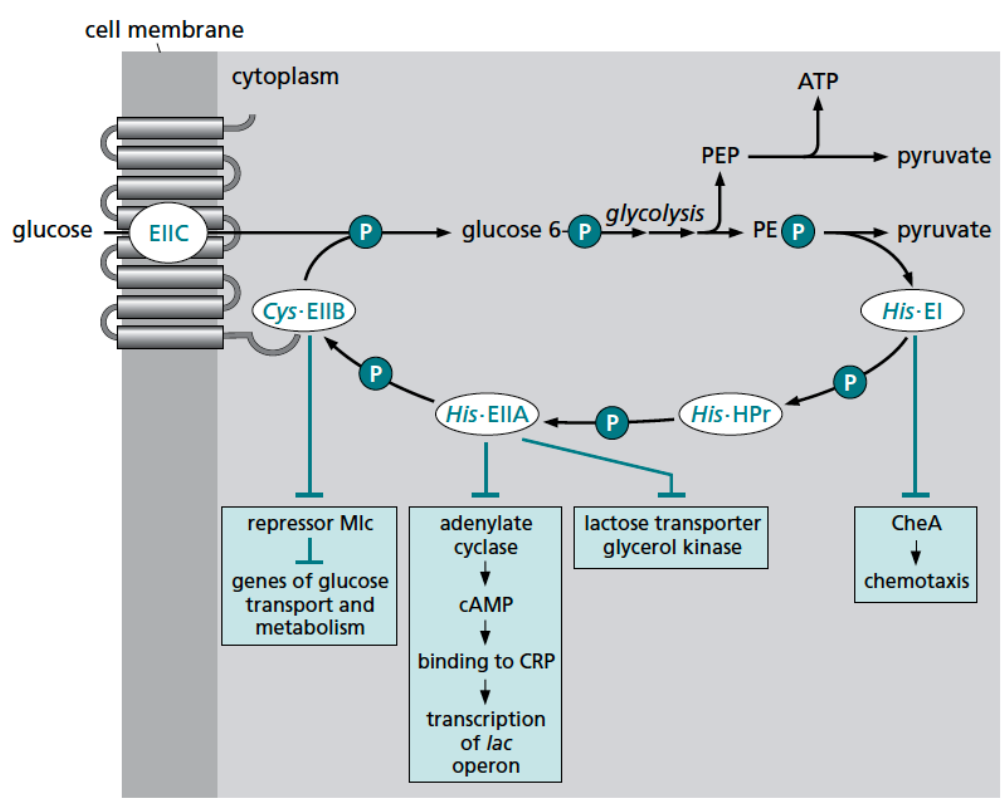

Figure 2. The phosphotransferase system (PTS) of E. coli. P is the phosphate residue. EI, $\mathrm{HPr}$, and EIIA are the cytoplasmatic proteins involved in the stepwise transfer of $\mathrm{P}$ from PEP to the membrane-bound channel complex EIIB/EIIC, where EIIB phosphorylates a sugar molecule that crosses the membrane and enters the cytoplasm through EIIC. Control mechanisms that operate based on the measurement of the states of EI, EllA and EIIB are represented in the square boxes. (Reproduced from Marks, Klingmüller, \& Müller-Decker, 2017, p. 96, with permission from Taylor and Francis Group LLC.)

The PTS is not itself a control mechanism. Each of its components performs an operation in the overall mechanism of extracting energy from glucose. At each step in the pathway, however, there is a control mechanism which makes a measurement of the phosphorylated state of the intermediate in the PTS pathway, a stand in for the availability of the relevant sugar, and couples that to an effector to regulate a cell process (for detailed reviews, see Deutscher et al., 2014; Västermark \& Saier, 2014). These control processes do not contribute to the activity of PTS pathway in phosphorylating a sugar molecule as it enters the cell. Nor are they directly involved in producing any other metabolic product. They are decoupled from the ongoing metabolic process, taking measurements from the state of the PTS pathway and, in response to the measurement, acting on other mechanisms.

The first two components, El and HPr, are common to the PTS pathways for all sugars. Accordingly, detecting when they are in the phosphorylated state provides a basis for measuring when no sugar of any type is available. This measurement can then be used to effect change in mechanisms needed in the absence of sugar in the bacterium's environment. Thus, the phosphorylation state of El is used to regulate flagellum movement by downregulating the autokinase activity of the sensor kinase CheA in the chemotaxis system (discussed above) when 
sugar is available. ${ }^{6}$ Unphosphorylated CheA cannot phosphorylate CheY, and unphosphorylated CheY cannot bind the motor. As a result, the motor rotates counterclockwise and the bacterium continues to swim, an appropriate response when the bacterium is continuing to find sugar. When sugar is not available and El is phosphorylated, the autokinase activity of CheA is upregulated, leading the bacterium to tumble before setting out in a new direction.

A second control process measures the phosphorylation of the second component of the PTS pathway, HPr. The conformation of HPr changes depending on whether it is phosphorylated. The conformation generated when $\mathrm{HPr}$ is unphosphorylated opens the binding site for glycogen phosphorylase to bind, thereby sequestering it and preventing it from catalyzing any reactions. The alternative conformation state generated when HPr is phosphorylated causes the glycogen phosphorylase binding site to release it. Glycogen phosphorylase is then free to catalyze the breakdown of glycogen to glucose, providing an energy source to the cell when no sugar is available to be metabolized.

Both of these control mechanisms are able to measure the phosphorylation state of PTS intermediates and produce effects on constraints in other mechanisms as a result of those measurements. They do not figure in the PTS pathway or in the mechanism over which they exercise control. They are decoupled from those processes and free to perform their control function based on the measurements made.

This is also true of control processes linked to the sugar-specific Ell processes. Being sugarspecific, the Ell complexes provide the basis for measuring the presence or absence of a given sugar in the bacterium's environment. We discuss just the members of the complex for glucose, which is the sugar preferred by E. coli. Adenyl cyclase is responsive to, and so measures, the phosphorylation state of EIIA ${ }^{\text {Glc }}$. When EIIA ${ }^{\text {Glc }}$ is phosphorylated, adenyl cyclase catalyzes the synthesis of cyclic AMP (cAMP) by removing a pyrophosphate $\left(\mathrm{P}_{2} \mathrm{O}_{5}{ }^{4-}\right)$ from ATP. cAMP then binds to the CAMP receptor protein ( $\mathrm{Crp})$, with the complex then serving as a transcription activator for numerous operons, including the lac operon that synthesizes the proteins needed to metabolize lactose when glucose is not available. (The lac operon also requires release from the inhibition by the lac-repressor, registering the availability of lactose). The phosphorylation state of ElIA $A^{G l c}$ is also measured by the transporters of numerous other sugars including lactose, maltose, galactose, and arabinose, inhibiting them when EIIA ${ }^{\text {Glc }}$ is unphosphorylated and releasing them from inhibition when EIIA ${ }^{\text {Glc }}$ is phosphorylated. Accordingly, these transporters do not transfer their respective sugars when glucose, the preferred sugar, is available. These control mechanisms, like those involving El and HPr, make measurements -in these cases, of the phosphorylation state of EIIA ${ }^{G l c}$, and operate on flexible constraints of other mechanisms based on that measurement. ${ }^{7}$

\footnotetext{
${ }^{6}$ This action on CheA is independent of the action of the MCPs. We discuss the importance of integrating multiple control processes through independent effects on common components in the next section.

${ }^{7}$ The last component in the PTS contributes to control in a somewhat different way. When ElIB ${ }^{\text {Glc }}$ is actively transporting glucose into the cell, it sequesters Mlc (Making large colonies protein). When not sequestered, Mlc represses both itself and a host of genes required for glucose transport and metabolism. By sequestering Mlc when
} 
In this section we have described several control mechanisms in E. coli that each takes advantage of materials made available by a common metabolic mechanism in the bacterium, the PTS pathway. Each involves measuring the phosphorylation state of an intermediate in the pathway that serves as a proxy for the presence of sugars in the bacterium's environment. This measurement is then the basis for an action on a flexible constraint in another of the bacterium's mechanisms. Each control mechanism, however, is decoupled from the ongoing metabolism. Its response is determined by the ability of the sensor to measure a specific variable. In that way, the control processes are decoupled from ongoing metabolic processes even as they are dependent on them.

\section{Opening and filling a signaling gap between sensors and effectors}

One consequence of decoupling, that is, freeing the components in a control mechanism from a role in ongoing metabolism, is that control processes can gain in complexity independently of any changes in metabolic mechanisms. One way it can increase in complexity is by adding components between the sensor and effector. In the simplest case of control mechanism, control through an allosteric enzyme, the sensor and effector components of control process and tightly linked as they belong to the same molecule-the conformation change resulting from the measurement by the sensor causes changes in the catalytic site of the enzyme. Even in that case the state of the sensor must be transmitted to the effector, but it is possible for transmission processes to be extended so as to involve multiple steps: the sensor can alter the conformation of another molecule that in turn acts to set the constraints in the effector component. Moreover, it is possible for a complex network of causal processes to intervene between sensors and effectors.

The process intervening between sensor and effector is often characterized as signaling. Signaling pathways can be very short. Control mechanisms in which the signal involves a conformation change within one molecule are referred to as one-component signaling systems. They are very common in bacteria (Ulrich, Koonin, \& Zhulin, 2005). Nonetheless, there are also numerous instances of two component signaling systems in which different molecules serve as the sensor and effector and a signal must be sent between them. Such an arrangement is useful when, as in the case of chemotaxis, the locations at which the sensor makes its measurement and at which the effector must operate on the flexible constraints of another mechanism are far apart. In many cases, the sensor protein is incorporated into a membrane whereas the effector is in the cytoplasm, and control requires a signal to be conveyed between the two.

As in the case of chemotaxis, the gap between the sensor and effector is often mediated by a kinase phosphorylating a signaling protein at a specific residue by hydrolyzing ATP or GTP. In bacteria most kinases add the phosphate to a histidine whereas in eukaryotes most kinases add phosphates to a serine, threonine, or tyrosine. In some cases, the protein that is

there is glucose for EIIBC to transport, this control mechanism prevents Mlc from inhibiting the transcription of these genes and allows for synthesizing more of the proteins needed to metabolize glucose (Nam et al., 2001). 
phosphorylated is the kinase itself (such molecules are known as autokinases). Yet it can equally well be a separate molecule that initiates changes along a pathway in which each molecule acts as a kinase phosphorylating the next.

The imposition of a signal between the measurement of the sensor and the action of the effector allows the same signal to produce effects on multiple effectors. This is illustrated in $E$. coli's system for maintaining osmotic pressure in response to changing osmolarity in its environment. In conditions of high osmolarity, the autokinase EnvZ phosphorylates itself at His243. The phosphate is then transferred to the Asp55 residue on the response regulator protein OmpR. OmpR acts as a transcription factor for two different proteins, downregulating expression of OmpF, which creates larger pores that enable more molecules to diffuse across the membrane, and upregulating $\mathrm{OmpC}$, which creates smaller pores. Both EnvZ and OmpR are autophosphatases; once they are phosphorylated at a given residue, they catalyze the removal of the phosphate. This restores the proteins to a condition in which they can initiate new responses whenever there is another change in osmolarity.

In addition to adding or removing a phosphate from a residue on a protein, signaling sometimes involves the synthesis (and subsequent degradation) of a small molecule that can then diffuse through the cell, potentially carrying a signal to multiple recipients. These small molecules are often referred to as second messengers, a designation that was adopted to distinguish them from intercellular signaling molecules such as hormones and neurotransmitters employed in multicellular organisms that had been discovered earlier. Sutherland and Rall (1958) discovered the first second messenger, CAMP, in eukaryotes. It was subsequently recognized as widely employed in prokaryotes. In E. coli, however, it only figures in the control mechanism described in the previous section, in which it is synthesized by adenylyl cyclase when ElIA ${ }^{\text {Glc }}$ is phosphorylated. It then activates transcription factors that act on a variety of operons.

In addition to allowing one measurement to act through multiple effectors, the inclusion of intermediates allows one effector to respond to multiple sensors measuring different variables. Another second messenger employed by E. coli both illustrates how such messengers can produce widespread effects and how they can be produced by multiple sensors. Guanosine tetraphosphate (ppGpp) figures in the stringent response. ppGpp is known as an alarmone since, in conditions in which the bacterium is running desperately short of resources to synthesize new constituents, it binds to any RNA polymerase it encounters, inhibiting it and thereby inhibiting synthesis of ribosomal RNA, ribosomal proteins, translation factors, and tRNA. It thereby effectively shuts down protein synthesis. ppGpp is also synthesized by two different sensors: RelA and SpoT. RelA is released from the ribosome when unbound tRNAs accumulate because they fail to find amino acids to which to bind. Once free, it functions first as a guanylate kinase that removes a pyrophosphate from ATP and adds it to GTP, yielding the pentaphosphate ppGppp, and then as a hydrolase to dephosphorylate ppGppp to ppGpp. SpoT functions similarly as a sensor detecting a shortage of multiple amino acids, lipids, phosphates, or iron to initiate the formation of ppGpp (Boutte \& Crosson, 2013). Either sensor can thus initiate the shutdown of protein synthesis. 
In this section we described how sensor and effector processes can be separated from each other, opening a gap to be filled by signaling processes involving, in the examples presented, a phosphorylated protein or a second messenger. This allows multiple sensors to make different measurements and yet produce the same signal or for the same signal to operate through different effectors to control different responses. When multiple sensors produce the same signal, the effector can carry out its control activity under a variety of detected conditions. By allowing multiple effectors to respond to the same signal, the control mechanism can bring about a coordinated alteration in the operation of multiple mechanisms. This arrangement can be further complicated so that an effector responds to a combination of signals - generating a response when any of several signals is received, operating like an OR-gate, when all of a set of signals is received, operating like an AND-gate, or according to another Boolean function.

The opening of a gap between sensor and effector results in control mechanisms that can respond to multiple conditions with integrated and adaptive responses. In principle, organisms could evolve an array of such control mechanisms that operate independently of each other. A further level of complexity arises when the same signaling components are employed by multiple control mechanisms. This results in what is known as crosstalk. As developed in the next section, crosstalk both enables more opportunities for coordination but also presents new challenges in segregating different responses appropriate for different conditions.

\section{Crosstalk: Integration and segregation}

We have already seen one example of crosstalk. In the chemotaxis system, CheA is phosphorylated by the MCPs. But it is also phosphorylated by El in the PTS systems. This case is not exceptional. The potential for crosstalk arises any time the same entities are employed in different control processes. As the term is used in engineering, crosstalk is usually regarded as undesirable since it undermines the ability to segregate different activities. That can be a problem in biology as well, but in many cases it can also be advantageous -it can enable the organism to integrate its responses to the particular combination of conditions it confronts. Segregation and integration are both important features of control processes. But they also compete-the more crosstalk there is in a system, the easier it is to integrate multiple responses, but the harder it is to segregate responses so that those not appropriate in a given circumstance do not occur. The investigation of how control processes both integrate and segregate is in its infancy. In this section we discuss investigations of control processes in $E$. coli that reveal ways in which this tradeoff is managed. We begin by focusing on how shared components foster integration and then consider how, despite this, individual control processes can be kept segregated.

The integration of multiple control processes is illustrated in control over the boundary between a bacterium and its environment. As we noted earlier, E. coli, like other gram-negative bacteria, has two cell membranes, an inner and an outer membrane. Between these is a region known as the envelop. Within the envelop resides the highly dynamic peptidoglycan cell wall which is constantly being modified over the lifespan of the bacterium (for a detailed discussion, see Deliagina, Musienko, \& Zelenin, 2019). The envelop is important for protecting the 
bacterium from external assaults, including from antibiotics (both those manufactured by humans and those produced by other bacteria). In order for the envelop to continue to protect the bacterium after an assault, it must detect damage and initiate appropriate repairs. For this it relies on numerous specialized control processes, each of which is capable of acting on flexible constraints in a mechanism that can execute a repair. They also share several small RNAs (sRNAs), short chains (50-250 nucleotides) of non-coding RNA, each of which can pair with multiple mRNA transcripts and affect their stability or translation. These sRNAs serve to integrate the responses of different control mechanisms. We start with two damage response mechanisms, the $\sigma^{\mathrm{E}}$ envelop stress response ${ }^{8}$ and the $\mathrm{Cpx}$ signaling pathway. We then describe instances of crosstalk between these mechanisms and how they enable integration of control processes and, by extension, the mechanisms they control.

The $\sigma^{\mathrm{E}}$ response (Figure $3 \mathrm{~A}$ ) is triggered by unfolding of outer membrane proteins and alterations in the outer membrane lipopolysaccharide. Under non-stress conditions, the inner membrane anti- $\sigma$ factor RseA sequesters $\sigma^{\mathrm{E}}$ at the plasma membrane. RseA is in turn protected by another protein, RseB, binding to it. Two preliminary steps are required to release $\sigma^{\mathrm{E}}$ from RseA. First, unfolded outer membrane proteins must bind to DegS, activating it. Second, lipopolysaccharide must bind RseB, removing it from RseA. After these preliminaries, DegS cleaves RseA, releasing $\sigma^{\mathrm{E}}$. ClpXP completes the process by releasing $\sigma^{\mathrm{E}}$ into the cytosol, where it acts as a signal to effectors that promote transcription of outer membrane protein chaperons, the Barn complex, and other genes that perform repair operations on the outer membrane.

(A)

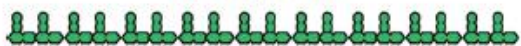
00000000000000000000000000000000000

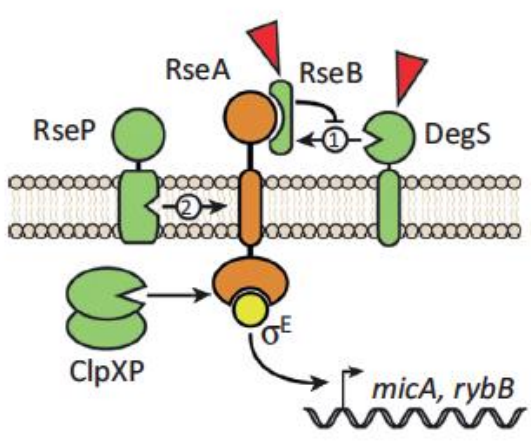

(B)

Cpx response

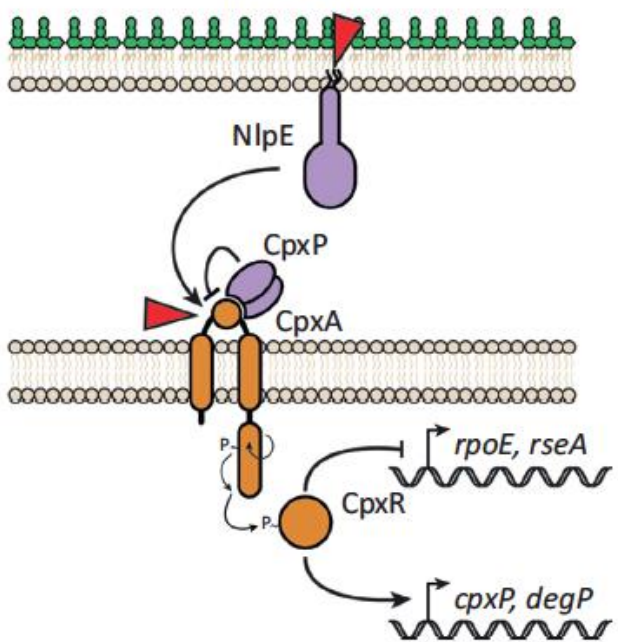

Figure 3. Two damage response mechanism of E. coli. The $\sigma^{E}$ envelop stress response (A) and $C p x$ pathway (B). The two mechanisms are interconnected because the $C p x$ effector protein CpxR activates the transcription of the $r p o E$ gene (B), which is responsible for

\footnotetext{
${ }^{8} \sigma$-factors (also designated as rpo for RNA polymerase) are proteins that bind to RNA-polymerase to initiate transcription in bacteria. $\sigma^{A}$ is known as the housekeeping $\sigma$-factor as it is involved in transcription of most genes that keep cells functioning. What are known as alternative $\sigma$-factors figure in transcription of genes needed for specific functions. $E$-coli has six alternative $\sigma$-factors. $\sigma^{\mathrm{E}}$ is involved in responses to exocytoplasmic stressors, including heat.
} 
the production of $\sigma^{\mathrm{E}}$, a signal component of the $\sigma^{\mathrm{E}}$ response $(\mathrm{A})$. From (Grabowicz \& Silhavy, 2017). Reproduced with permission from Elsevier.

The Cpx envelop stress response is a typical two component system (Figure 3B). CpxA is an inner membrane histidine kinase that phosphorylates itself in response to a range of conditions that induce protein misfolding in inner membrane and periplasmic proteins, including elevated $\mathrm{pH}$, high salt concentrations, alterations in IM lipid composition, and defects in the cell wall. Phosphorylated CpxA then transfers its phosphate to the CpxR receiver domain. CpxR is the effector, acting as a transcription factor on numerous genes involved in repair functions (e.g., $m d t A$, which codes for a protein involved in removal of $\beta$-lactamic antibiotics from the cell and $d s b A$ and $\operatorname{deg} P$, which encode periplasmic foldases and chaperons).

To this point we have described the two control mechanisms as operating independently. But they also share components. One of these is represented in Figure 3, but disguised by the use of different labels: $r p o E$ is the gene for $\sigma^{E}$. Thus, among the responses initiated by CpxR is transcription of $\sigma^{\mathrm{E}}$, the signaling component in the $\sigma^{\mathrm{E}}$ response. Figure 4 represents other modes of interaction between these and other control mechanisms serving to regulate the envelop. For example, MicA, whose transcription is regulated by $\sigma^{\mathrm{E}}$, regulates $p h o P Q$ transcription, which encodes a gene involved in an additional stress response that acts on the lipopolysaccharide of the outer membranes. Several of the components in the CpxR mechanism-OmrA, OmrB, and MicF-are also components of the EnvZ/OmpR regulon, discussed in the previous section as regulating osmotic pressure in the envelop. ${ }^{9}$ Also shown in Figure 4 is an interaction between the $C p x$ response system and another stress response system, the Rcs system: both systems promote the transcription of the sRNA RprA, which not only inhibits the Cpx system but promotes the expression of $\sigma^{S}$, the master stress regulator which figures in the transition from solitary life to incorporation in a biofilm. ${ }^{10}$ Beyond providing a detailed review of these and yet other interconnections of the stress response systems, Grabowicz and Silhavy (2017) offer hypotheses about how they facilitate coordinated responses to various challenges to the envelop. They conclude:

During transitions between ecological niches or during infection, bacteria encounter continuous, complex environmental changes that could trigger multiple stress inputs and it remains to be seen how cells interpret these complex signals to mount a coherent response against this onslaught. The ESRs RNAs have clear functions in regulating effectors as well as feedback functions in regulating responses. Notably, the sRNAs

\footnotetext{
${ }^{9}$ Of particular note is MicF, which not only inhibits the EnvZ/OmpR regulon but also the the CpxA-CpxR response system responsible for its own activation. In characterizing MicF, Grabowicz and Silhavy (2017) comment "MicF is remarkable for establishing a negative-feedback loop that is wired through two signal transduction systems" ( $p$. 236).

${ }^{10}$ We have focused in this paper on control processes operative in E. coli when they act as solitary individuals. But they are also able to form biofilms in which they create an environment in which individual bacteria are protected from a variety of external threats. This requires a large-scale lifestyle change in each bacterium ceases to use its flagella to locomote and deploy production mechanisms that attach to a surface and generate an extracellular matrix that consists of proteins, nucleic acids, lipids and phospholipids, colanic acid, and adhesins. The transition between lifestyles involves interactions between a host of control mechanisms (for overviews, see Sharma et al., 2016; Markova, Anganova, Turskaya, Bybin, \& Savilov, 2018).
} 
appear to provide numerous avenues for inter-ESR signaling, including by controlling transcripts specifying proteins at the apex of stress signaling circuits (Figure 4). We suggest that SRNAs will prove to have important roles in coordinating the highly interconnected stress responsive network (p. 240).

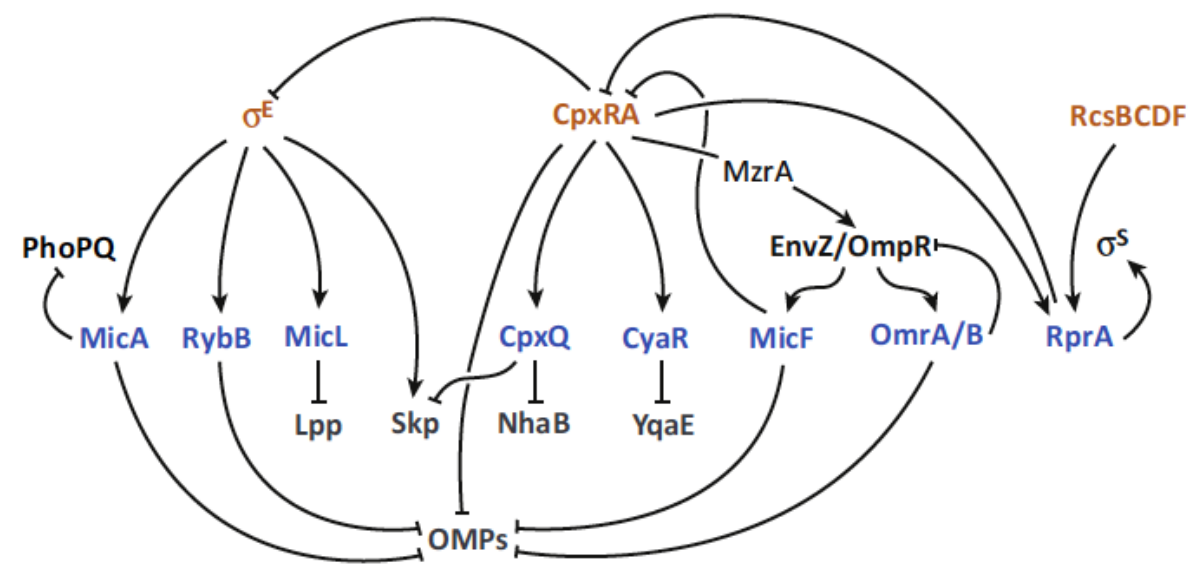

Figure 4. Interconnections between several control mechanisms of $E$. coli by direct interaction and by means of short RNAs (in blue). Stress response mechanisms are in orange. Proteins involved in other control mechanisms are in black. From (Grabowicz \& Silhavy, 2017). Reproduced with permission from Elsevier.

This example identifies ways in which control mechanisms are integrated by starting with details of what is known about individual responses and identifying common components. A different strategy starts by first identifying all proteins capable of bonding to each other (in what are known as protein-protein interactions) and focusing on those associated with a given phenomenon. Typas and Sourjik (2015) analyzed interactions between proteins involved in chemotaxis and flagella activity. They applied clustering techniques to identify three clusters of proteins. By using what is known about the cellular functions of individual proteins, they associated two of the clusters with mechanisms involved in the operation of the flagellum and one with the chemotaxis control process (Figure 5). There is only one connection between the chemotaxis system and the rotary engine and switch complex, that between CheY and FliM (which we discussed above). However, in addition to extensive interaction between components of the two flagella mechanisms, there are numerous protein-protein interactions between proteins in the clusters and those shown as outside the clusters (these would usually appear in other clusters if the broader network were represented). These are instances of crosstalk. Crr and Ptsl integrate signals from the phosphotransferase system (discussed above) with two components of the chemotaxis system, CheW and CheA. Another example of crosstalk involves high temperature protein $\mathrm{G}(\mathrm{HtpG})$, a heat shock protein that serves as a chaperon in protein folding: it interacts with proteins in both mechanisms and with CheA. Finally, the flagellum itself interacts not just with the chemotaxis system, but with H-NS (involved in global gene regulation), FrdA (involved in energy metabolism), and YcgR (linked with c-di-GMP second-messenger signaling). 


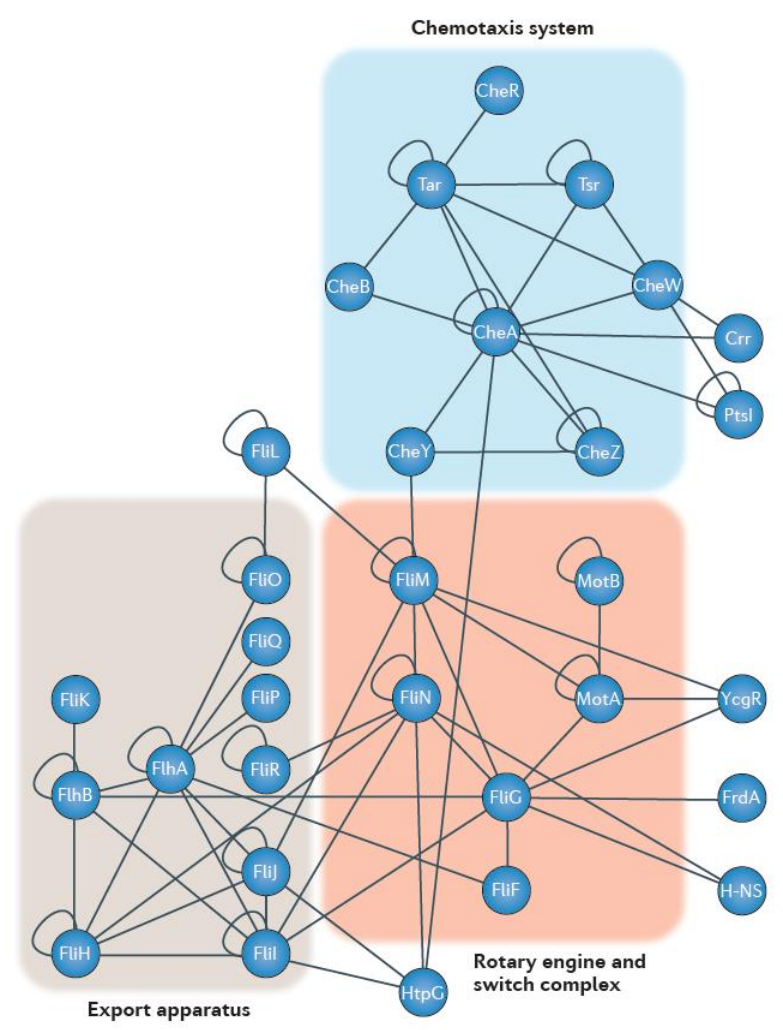

Figure 5. Three modules, shown in color in an E. coli PPI network. Two correspond to flagella production mechanisms and one to chemotactic control. Several other proteins, involved in other control processes, are shown outside the modules. There is extensive crosstalk between the modules and between components of the modules and other proteins involved in control. From Typas and Sourjik (2015). Reproduced with permission from Springer Nature.

These examples illustrate the widespread crosstalk between control mechanisms and other mechanisms carrying out activities in E. coli. This crosstalk is assumed to be extremely useful in enabling $E$. coli to integrate their various responses to the challenges they confront. But, as we noted, this means of coordination comes at a cost of compromising the ability of the bacterium to segregate its responses so that it can activate one production mechanism without activating others that are not required or counter-indicated in a given situation. How, in the face of crosstalk, is it possible to segregate responses so that only those required in a specific situation are actually carried out? One way to conceptualize the issue is that PPI networks are partially constructed from techniques such as yeast 2 hybrid screens which show whether it is possible for two proteins to bind. These screens do not show whether they actually bind in the cell. One way to prevent undesired crosstalk is to keep proteins that can interact separated from one another, functionally or spatially, when interaction is not desired.

We focus on spatial segregation. Eukaryotic cells encapsulate proteins in membrane-bound organelles that prevent diffusion. Thus, hydrolytic enzymes that would break down proteins are kept separated in the lysosome so that they only act on proteins selected for break down. This 
solution was not thought to be available to bacteria. Indeed, their cytoplasm is often viewed as a homogeneous milieu in which any constituent can diffuse everywhere in the cell. In recent years, however, researchers have discovered a variety of ways in which the bacterial cytoplasm can be differentiated into different environments in which operations, including control operations, can be segregated. We note three. ${ }^{11}$

First, intracellular membranes and vesicles, which are thought to be derived from invagination of the plasma membrane, occur frequently in bacteria, including E. coli (Bogdanov, Aboulwafa, \& Saier Jr, 2013). Proteins, including those figuring in control, are often positioned structurally vis a vis one another in membranes. This enables them to selectively bind to those bound to the same membrane but not with those not so bound.

The second mode of segregation is an extension of this - often a protein serves as a scaffold to which other selected proteins can bind, creating a local environment in which only specific proteins interact. One context is in cell division, which in E. coli begins with the formation of a Z-ring around the middle of the cell. The primary constituent of the ring is FtsZ; FtsZ molecules polymerize end to end to form the ring around the circumference of the cell. The FtsZ ring provides a scaffold to which specific other proteins attach. Some of these contribute to the construction of a cell wall between daughter cells, but others serve to regulate these activities. As a result of the arrangement in which they bind to FtsZ, some actions are facilitated, and other possible interactions are prevented from occurring (Lutkenhaus \& Du, 2017).

Third, researchers have recently found evidence of condensates resulting from the separation of phases within liquids (liquid-liquid phase separation). The phase separation is brought about through weak protein-nucleic acid and protein-protein interactions involving intrinsically disorganized regions of the proteins. The condensates are known as membraneless organelles. Since they do not have a membrane barrier, molecules can diffuse in and out of the condensates, but they typically do so slowly, creating differentiated environments that favor specific molecular interactions. ${ }^{12}$ Membraneless organelles have mostly been studied in eukaryotic cells, as the small size of bacterial cells makes studying them in bacteria much more challenging. Nonetheless Azaldegui, Vecchiarelli, and Biteen (2021) summarize suggestive evidence for several membraneless organelles occupying different regions in $E$. coli and other bacteria. One involves the control over FtsZ polymerization discussed in the previous paragraph. Monterroso et al. (2019) present evidence that when SImA binds to FtsZ, they together generate a membraneless organelle that sequesters FtsZ near the membrane and away from DNA-rich regions until it is recruited to mid-cell at the initiation of cell division.

In this section we have shown not only how crosstalk can lead to integration of control process, creating the potential for coherent action, but also the need for segregation so that control

\footnotetext{
${ }^{11}$ Ross (2018) discusses segregation of substrates (or 'starting materials') in the context of what we refer to as 'production mechanisms'.

12 Membraneless organelles are much more dynamic than those with membranes as they can form and dissipate without constructing lipid membranes. This is a factor that has motivated recent interest in them.
} 
processes can result in specific responses to distinct situations. The processes we have identified as responsible for integration and segregation are likely to be just exemplars of broader classes of processes. Discovery of crosstalk comes as researchers identify components involved in different control processes and then recognize the same component showing up in different instances. Once the possibility for crosstalk is identified, it remains to determine whether it is actual and contributes to the integrated response capacities of the organism or whether it is prevented, perhaps by specialized processes that impose segregation.

\section{How to understand control mechanisms}

Our goal in this paper has been to identify key elements of control mechanisms that enable organisms to produce integrated and adaptive behavior. We first extracted two fundamental features: (1) the need for sensors that measure values of variables and effectors that produce change in soft constraints in other mechanisms that are (2) decoupled from ongoing metabolism so as to exercise control based on the measurements made. Although sensors and effectors can be directly connected, we described how intermediaries can transmit signals between them and how such intermediaries allow for coordinated responses within and between control mechanisms. We noted the challenge that enabling distinct responses to different conditions requires countering the tendency for integration with strategies that segregate responses.

Although we drew our analysis from examination of control mechanisms in $E$. coli, we urge that these features are likely to be common to control mechanisms in biology. As an indication that this might be the case, we very briefly show how these characteristics are exemplified in control processes in multicellular animals. Although a significant amount of control in animals is achieved chemically through the endocrine system, neurons play a central role. Neurons are effectors of control by releasing chemicals that act on other cells, including secretory and muscle cells. They act by binding to receptors on the cell surface and either, in the case of ionotropic receptors, directly generating an action potential in the postsynaptic cells or, in the case of metabotropic receptors, initiating chemical activities within the cell that alter the operation of various intracellular mechanisms. Within neurons, the activity of effectors is a response to sensors that respond to internal or external states by initiating electrical activity that is transmitted to the effectors. These activities of neurons are dependent upon the metabolic mechanisms of organisms but are decoupled from them so that the activities of effectors are responsive to the measurements made by sensors.

In many cases, the link between sensors and effectors involves multiple neurons connected by synapses. This is one factor that generates the potential for crosstalk. While there can be oneto-one mappings between pre- and post-synaptic neurons, often multiple upstream neurons converge on one neuron and each neuron sends outputs to multiple downstream neurons. Moreover, these inputs and outputs can take many forms given the diversity of neurotransmitters. In addition to transmitters such as glutamate and GABA that are released and taken up at individual synapses, there are monoamine and neuropeptide transmitters that are released at multiple locations on neurons, including on dendrites, and diffuse broadly 
through extracellular fluids. These transmitters often modulate the responses of other receptors to more traditional transmitters (Katz, 1999). As exemplified in subcortical nuclei, there is a great deal of crosstalk between neurons, enabling highly integrated responses. ${ }^{13}$ This gives rise to the same challenge as we observed in bacteria-sufficiently segregating neural activity so that control can give rise to specific actions. One common strategy for segregating responses is to establish competition between responses in which one alternative is pursued while others are suppressed (the basal ganglia are organized into a structure that appears to support such competition). We suggest that the same principles we extracted from $E$. coli are manifest in control exercised in all organisms. Individual control mechanisms enable the organism to utilize mechanisms involved in metabolism and movement in ways appropriate to circumstances, and the manner in which these are integrated and segregated accounts for the integrated and adaptive behavior of the whole organism.

As we have argued previously (Bich \& Bechtel, 2021), control mechanisms provide a bridge for relating the understanding of organisms provided by the mechanist tradition and by autonomy theorists. Many of the mechanisms constituting an organism carry out specific productive tasks that are required for an organism to build, maintain, and repair itself. Understanding their operation, however, does not explain the integrated and adaptive manner in which organisms perform these activities. Control mechanisms enable these mechanisms to be called upon when conditions measured by the control mechanisms indicates they are needed. The shared components in these control mechanisms results in the ability to integrate calls upon the mechanisms performing the productive tasks on which organisms depend.

We have differentiated control mechanisms from other mechanisms in virtue of their ability to measure variables and use the results of such measurement to alter constraints in other mechanisms. In discussing control, though, we have had occasion to invoke other terms, including pathway and network. Some philosophers of science have argued for differentiating explanations that employ these terms from mechanistic explanations. Ross (2021), for example, argues for differentiating pathways from mechanisms, contending that "these concepts: (a) have unique features, (b) that they are associated with distinct strategies of causal investigation, and (c) that they figure in importantly different types of explanation." Pathway explanations, she argues, emphasize the sequence of causal steps that transfer a conserved substance from one to the next, while often abstracting from causal detail. One can make a similar argument about network accounts - the networks represented in Figures 4 and 5 also abstract from causal detail (the network diagram in Figure 4 does indicate directionality of effects and whether each is excitatory or inhibitory; even that is left out in Figure 5). In contrast, according to Ross explanations in terms of mechanisms place "emphasis on the 'force,' 'action,' and 'motion' involved in causal relationships."

\footnotetext{
13 This is illustrated by the difficulty of specifying just one control activity for a given nucleus in the hypothalamus. Hypocretin neurons receive signals from sensors reporting on many different conditions facing the organism and produce effects on multiple activities, including feeding and overall state of awakeness.
} 
Before focusing on how they relate to mechanistic explanations, we first review how networks and pathways entered into the examples discussed. Our first example of a control process, chemotaxis, involved a pathway of phosphorylation steps from CheA to Che $Y$ to FliM. In eukaryotes there are phosphorylation pathways involving many more steps; the Ras pathway, for example, begins with a GTPase and proceeds through four kinases, each of which phosphorylates and thereby activates the next. Often as research unfolds, what was represented as a simple pathway is recognized to branch in multiple directions, morphing into network representations such as in Figure 4.

In deciding which concept is most appropriate to describe these control processes, a critical issue raised by Ross is the extent to which research on these processes in fact abstracts from causal details. In a phosphorylation pathway, one may simply describe each component as phosphorylating the next, but researchers often go a step further, describing a specific component as a kinase (or an autokinase) and specifying the site of phosphorylation. Likewise, in constructing a network one may abstract to different degrees. Figure 5 represents each protein as a node and each protein-protein interaction as an edge. Figure 4 is less abstract, indicating the direction of the effect of one component on the next and whether the effect is excitatory or inhibitory. In some cases, researchers abstract still less, using different glyphs to represent different types of entities or causal processes (Bechtel, 2020). ${ }^{14}$

A further consideration Ross invokes in distinguishing pathways from mechanisms is that mechanistic accounts are focused on local activities in the pathway, not at activity exhibited in the pathway as a whole. It is certainly true that researchers on pathways and networks often focus on low-level causal detail when they address what is happening at each node of a pathway or network. But mechanistic accounts are not limited to one level. Many mechanists have argued that mechanisms decompose into component mechanisms and component mechanisms compose into higher-level mechanisms. What is crucial to the composition of mechanisms into larger-scale mechanisms is the organization of the components. Pathway or network representations are used within mechanistic accounts to represent the organization of the components. Such representations, however, are static. The goal of a mechanistic explanation is to show how a given phenomenon results from the organization of the components. To go from a static representation of the components and their relations to each other to understanding the mechanism typically requires either the researcher mentally animating the mechanism (Hegarty, 1992) or developing a computational model (Brigandt, 2013; Bechtel \& Abrahamsen, 2010). Through these strategies researchers simulate how each component acts and follow out the effects of such action on other components to which it is connected in a pathway or a network. By following activity through the pathway or network,

\footnotetext{
${ }^{14}$ Network representations are often the product of extensive epistemic work. In constructing a network from protein-protein interactions, the initial representation is often what is characterized as a hairball-showing all the connections for each protein but without any order. Representations such as Figure 5 result from applying numerous analytical strategies, including annotating nodes with information that is known about the corresponding protein. The goal is to develop a representation that can then serve as a heuristic in creating new mechanistic understanding of the system being represented (Bechtel, 2019).
} 
such accounts characterize how the whole mechanism in which the pathway or network is situated produces its phenomenon.

We agree with Ross that scientists deploy distinctive reasoning in developing pathway and network representations. There may be contexts in which these are developed and used for explanatory purposes independently of advancing mechanistic explanations. But in the context of understanding control, we contend that pathways and networks are developed to relate the components of control mechanisms. ${ }^{15}$ When intermediates are included between sensors and effectors in control mechanisms, characterizing them as organized into a pathway provides a means of tracking how control is carried out. Likewise, when researchers identify crosstalk, characterizing the resulting network allows them to understand how control mechanisms operate together to produce controlled activity.

While we contend that characterizing control mechanisms is a productive way to understand how control is achieved in living organisms, we do not view mechanisms as differentiated in the world prior to scientific investigations. Rather, they are identified by scientists in the process of developing explanations-researchers select components and treat them as working together as a mechanism. Depending on how they characterize the phenomenon they are trying to explain and their explanatory objectives, different researchers treat different components as constituting a mechanism and others as part of the mechanism's environment (Bechtel, 2015). This is true of mechanisms carrying out the basic metabolic activities of organisms, but is especially true of control mechanisms that exhibit crosstalk. Representing crosstalk as a network reveals how integrated control processes in living organisms are. A network representation provides the equivalent of a roadmap, identifying routes through which control activities can travel. Given their explanatory objectives, researchers may identify specific pathways to incorporate into their account of a control mechanism - their explanation will then focus on activities occurring along these pathways, backgrounding others. Other researchers with different explanatory objectives may make different choices. ${ }^{16}$

\section{Conclusions}

Control mechanisms, we have argued, are what enable biological organisms to act as integrated wholes in versatile ways that enable them to maintain themselves as systems far from equilibrium with their environments. By focusing on examples of control mechanisms in a

\footnotetext{
${ }^{15}$ One concern Ross advances is that if mechanisms are understood as little more than componential systems, they are not differentiated from other systems and mechanistic explanation becomes an extremely vague concept. There are, it should be noted, other forms of explanation beyond ones that appeal to a system's components (Levy \& Bechtel, 2020). But Ross does make an important point, and indeed it is an important philosophical contribution to differentiate explanatory reasoning that appeals to pathways or networks. In some cases, it might be more productive not to differentiate a mode of explanation as different in kind from mechanistic ones, but to distinguish types of mechanistic explanation and develop a taxonomy of mechanistic/componential explanations. This might require multiple dimensions.

${ }^{16}$ This selectivity is illustrated in this paper. In different sections we discussed different control mechanisms only to reveal later how they are interconnected via crosstalk with other control mechanisms.
} 
relatively simple single-cell organism, $E$. coli, we have identified several important features of control. We have argued that control mechanisms must, at a minimum, contain sensors that measure the value of variables relevant to the appropriate deployment of other mechanisms and effectors that act on flexible constraints in these other mechanisms so that they operate when and as needed. By acting on these other mechanisms as a result of the measurements they make, control mechanisms are decoupled from basic metabolic processes of organisms. The connection between sensors and effectors can be direct-the structure of a protein can be altered when it binds and measures a variable and, as a result of the conformation it adopts, act upon another mechanism. But in many cases intermediate signals can intervene between the sensory and the effector. This enables organisms to respond to a combination of measurements with multiple responses. It also raises the possibility of crosstalk between different control mechanisms. This facilitates integrating the response of an organism, but it also presents a challenge of allowing for selective responses to specific conditions. We identified some ways in which this is accomplished in bacteria.

We propose that a focus on control mechanisms provides a bridge between theoretical frameworks of the new mechanists and autonomy theorists. Both can make important contributions-mechanists by characterizing the functioning of control mechanisms and revealing how they are investigated empirically and autonomy theorists by characterizing how responses tied to measurements enable organisms to maintain themselves. The account advanced here is just a start, and one that we expect can be productively elaborated on by examining other examples of control mechanisms in biology. One topic that requires much more exploration is how control mechanisms work together to maintain an organism. We have noted how, as a result of crosstalk, the activities of multiple control mechanisms are integrated and coordinated. But crosstalk could also lead to conflicts between behavioral responses. Humans often design institutions in which one person holds ultimate authority to resolve conflicts. In organisms such as E. coli, and we would argue in all organisms, there is not a central executive overseeing a hierarchy of control activities. Instead, multiple different control mechanisms each produce different effects while connected in multiple ways. Evolution may provide part of the answer as to how these different control mechanisms succeed in working together-when new control mechanisms fail to maintain the autonomy of the organism, the organism leaves few or no descendants (Freddolino \& Tavazoie, 2012). As a result, control mechanisms found in extant organisms are ones that integrate successfully. A further question is whether there are design principles that can enable investigators to make sense of how multiple control mechanisms together serve to maintain organisms. To the degree these can be identified, they may constitute the subject matter of investigations that bridge the mechanist and autonomy traditions.

Acknowledgements: The authors thank Lauren Ross and Jason Winning for the careful reading and useful comments on a previous version of this paper.

Funding: The authors acknowledge funding from the Basque Government (Project: IT1228-19 for LB), Ministerio de Ciencia, Innovación y Universidades, Spain (research project PID2019104576GB-I00 for LB and WB, and 'Ramon y Cajal' Programme RYC-2016-19798 for LB). 
Declaration of Conflicting Interests: The Authors declare that there is no conflict of interest

\section{References}

Azaldegui, C. A., Vecchiarelli, A. G., \& Biteen, J. S. (2021). The emergence of phase separation as an organizing principle in bacteria. Biophysical Journal, 120(7), 1123-1138.

doi:https://doi.org/10.1016/j.bpj.2020.09.023

Bechtel, W. (2015). Can mechanistic explanation be reconciled with scale-free constitution and dynamics? Studies in History and Philosophy of Science Part C: Studies in History and Philosophy of Biological and Biomedical Sciences, 53, 84-93. doi:http://dx.doi.org/10.1016/j.shpsc.2015.03.006

Bechtel, W. (2019). Analyzing network models to make discoveries about biological mechanisms. British Journal for the Philosophy of Science, 70, 459-484.

Bechtel, W. (2020). Data journeys beyond databases in systems biology: Cytoscape and NDEx. In S. Leonelli \& N. Tempini (Eds.), Data Journeys in the Sciences. Cham, Switzerland: Springer.

Bechtel, W., \& Abrahamsen, A. (2005). Explanation: A mechanist alternative. Studies in History and Philosophy of Biological and Biomedical Sciences, 36(2), 421-441.

Bechtel, W., \& Abrahamsen, A. (2010). Dynamic mechanistic explanation: Computational modeling of circadian rhythms as an exemplar for cognitive science. Studies in History and Philosophy of Science Part A, 41(3), 321-333.

Bechtel, W., \& Bich, L. (2021). Grounding cognition: heterarchical control mechanisms in biology. Philosophical Transactions of the Royal Society of London: B Biolological Sciences, 376(1820), 20190751. doi:10.1098/rstb.2019.0751

Bechtel, W., \& Richardson, R. C. (1993/2010). Discovering complexity: Decomposition and localization as strategies in scientific research. Cambridge, MA: MIT Press. 1993 edition published by Princeton University Press.

Bi, S., \& Sourjik, V. (2018). Stimulus sensing and signal processing in bacterial chemotaxis. Current Opinion in Microbiology, 45, 22-29. doi:https://doi.org/10.1016/j.mib.2018.02.002

Bich, L., \& Bechtel, W. (2021). Mechanism, autonomy, and biological explanation. Biology and Philosophy, 36(53). doi:10.1007/s10539-021-09829-8

Bich, L., \& Moreno, A. (2016). The role of regulation in the origin and synthetic modelling of minimal cognition. Biosystems, 148, 12-21. doi:10.1016/j.biosystems.2015.08.002

Bich, L., Mossio, M., Ruiz-Mirazo, K., \& Moreno, A. (2016). Biological regulation: controlling the system from within. Biology \& Philosophy, 31(2), 237-265. doi:10.1007/s10539-0159497-8

Bogdanov, M., Aboulwafa, M., \& Saier Jr, M. H. (2013). Subcellular Localization and Logistics of Integral Membrane Protein Biogenesis in $\langle\mathrm{b}\rangle\langle\mathrm{i}\rangle$ Escherichia coli $</ \mathrm{i}\rangle\langle/ \mathrm{b}\rangle$. Journal of Molecular Microbiology and Biotechnology, 23(1-2), 24-34.

Bourret, R. B., \& Stock, A. M. (2002). Molecular Information Processing: Lessons from Bacterial Chemotaxis. Journal of Biological Chemistry, 277(12), 9625-9628.

doi:10.1074/jbc.R100066200 
Boutte, C. C., \& Crosson, S. (2013). Bacterial lifestyle shapes stringent response activation. Trends in Microbiology, 21(4), 174-180. doi:https://doi.org/10.1016/j.tim.2013.01.002

Brigandt, I. (2013). Systems biology and the integration of mechanistic explanation and mathematical explanation. Studies in History and Philosophy of Biological and Biomedical Sciences, 44(4), 477-492. doi:10.1016/j.shpsc.2013.06.002

Craver, C. F., \& Darden, L. (2013). In search of mechanisms: Discoveries across the life sciences. Chicago: University of Chicago Press.

Deliagina, T. G., Musienko, P. E., \& Zelenin, P. V. (2019). Nervous mechanisms of locomotion in different directions. Current Opinion in Physiology, 8, 7-13. doi:https://doi.org/10.1016/j.cophys.2018.11.010

Deutscher, J., Aké, F. M. D., Derkaoui, M., Zébré, A. C., Cao, T. N., Bouraoui, H., . . Joyet, P. (2014). The Bacterial Phosphoenolpyruvate:Carbohydrate Phosphotransferase System: Regulation by Protein Phosphorylation and Phosphorylation-Dependent Protein-Protein Interactions. Microbiology and Molecular Biology Reviews, 78(2), 231. doi:10.1128/MMBR.00001-14

Deutscher, J., Francke, C., \& Postma, P. W. (2006). How phosphotransferase system-related protein phosphorylation regulates carbohydrate metabolism in bacteria. Microbiol Mol Biol Rev, 70(4), 939-1031. doi:10.1128/MMBR.00024-06

Dyer, C. M., Vartanian, A. S., Zhou, H., \& Dahlquist, F. W. (2009). A molecular mechanism of bacterial flagellar motor sbourwitching. Journal of Molecular Biology, 388(1), 71-84. doi:https://doi.org/10.1016/j.jmb.2009.02.004

Fechner, G. T. (1860). Elemente der Psychophysik. Leipzig: Breitkopf und Härtel.

Freddolino, P. L., \& Tavazoie, S. (2012). Beyond Homeostasis: A Predictive-Dynamic Framework for Understanding Cellular Behavior. Annual Review of Cell and Developmental Biology, 28(1), 363-384. doi:10.1146/annurev-cellbio-092910-154129

Glennan, S. (2017). The new mechanical philosophy. Oxford: Oxford University Press.

Grabowicz, M., \& Silhavy, T. J. (2017). Envelope Stress Responses: An Interconnected Safety Net. Trends in Biochemical Sciences, 42(3), 232-242. doi:10.1016/j.tibs.2016.10.002

Hegarty, M. (1992). Mental animation: Inferring motion from static displays of mechanical systems. Journal of Experimental Psychology: Learning, Memory, and Cognition, 18(5), 1084-1102.

Hooker, C. A. (2013). On the import of constraints in complex dynamical systems. Foundations of Science, 18(4), 757-780. doi:10.1007/s10699-012-9304-9

Katz, P. S. (1999). What are we talking about? Modes of neuronal communication. In P. S. Katz (Ed.), Beyond neurotransmission: Neuromodulation and its importance for information processing (pp. 1-28). New York: Oxford.

Kauffman, S. A. (2000). Investigations. Oxford: Oxford University Press.

Levy, A., \& Bechtel, W. (2020). Beyond machine-like mechanisms. In S. Holm \& M. Serban (Eds.), Philosophical perspectives on the engineering approach in biology: Living machines? New York: Routledge.

Lutkenhaus, J., \& Du, S. (2017). E. coli cell cycle machinery. In J. Löwe \& L. A. Amos (Eds.), Prokaryotic Cytoskeletons: Filamentous Protein Polymers Active in the Cytoplasm of Bacterial and Archaeal Cells (pp. 27-65). Cham: Springer International Publishing. 
Lyon, P. (2015). The cognitive cell: Bacterial behaviour reconsidered. Frontiers in Microbiology, 6. doi:10.3389/fmicb.2015.00264

Machamer, P., Darden, L., \& Craver, C. F. (2000). Thinking about mechanisms. Philosophy of Science, 67, 1-25.

Marken, R. S., \& Powers, W. T. (1989). Random-walk chemotaxis: trial and error as a control process. Behavioral Neuroscience, 103(6), 1348-1355. doi:10.1037//07357044.103.6.1348

Markova, J. A., Anganova, E. V., Turskaya, A. L., Bybin, V. A., \& Savilov, E. D. (2018). Regulation of Escherichia coli Biofilm Formation (Review). Applied Biochemistry and Microbiology, 54(1), 1-11. doi:10.1134/S0003683818010040

Marks, F., Klingmüller, U., \& Müller-Decker, K. (2017). Cellular signal processing: An introduction to the molecular mechanisms of signal transduction (Second edition. ed.).

Maturana, H. R., \& Varela, F. J. (1980). Autopoiesis: The organization of the living. In H. R. Maturana \& F. J. Varela (Eds.), Autopoiesis and cognition: The realization of the living (pp. 73-138). Dordrecht: Reidel.

Militello, G., \& Moreno, A. (2018). Structural and organisational conditions for being a machine. Biology \& Philosophy, 33, 35. doi:10.1007/s10539-018-9645-z

Monterroso, B., Zorrilla, S., Sobrinos-Sanguino, M., Robles-Ramos, M. A., Lopez-Alvarez, M., Margolin, W., ... Rivas, G. (2019). Bacterial FtsZ protein forms phase-separated condensates with its nucleoid-associated inhibitor SImA. EMBO Rep, 20(1). doi:10.15252/embr.201845946

Moreno, A., \& Mossio, M. (2015). Biological autonomy: A philosophical and theoretical inquiry. Dordrecht: Springer.

Nam, T.-W., Cho, S.-H., Shin, D., Kim, J.-H., Jeong, J.-Y., Lee, J.-H., . . Seok, Y.-J. (2001). The Escherichia coli glucose transporter enzyme IICBGlc recruits the global repressor Mlc. The EMBO Journal, 20(3), 491-498. doi:https://doi.org/10.1093/emboj/20.3.491

Pattee, H. H. (1973/2012). The physical basis and origin of hierarchical control. In Laws, language and life (Vol. 7, pp. 91-110): Springer Netherlands.

Powers, W. T. (1990). Control theory: A model of organisms. System Dynamics Review, 6(1), 120. doi:https://doi.org/10.1002/sdr.4260060102

Rosen, R. (1970). Dynamical system theory in biology: Stability Theory and Its Applications. New York: Wiley-Interscience.

Ross, L. N. (2018). Causal Selection and the Pathway Concept. Philosophy of Science, 85(4), 551572. doi:10.1086/699022

Ross, L. N. (2021). Causal concepts in biology: How pathways differ from mechanisms and why it matters. The British Journal for the Philosophy of Science, 72(1), 131-158. doi:10.1093/bjps/axy078

Ross, L. N. (in press). Causal control: A rationale for causal selection. In Causation in Biology. Minneapolis: University of Minnesota Press.

Sharma, G., Sharma, S., Sharma, P., Chandola, D., Dang, S., Gupta, S., \& Gabrani, R. (2016). Escherichia coli biofilm: development and therapeutic strategies. Journal of Applied Microbiology, 121(2), 309-319. doi:https://doi.org/10.1111/jam.13078

Sourjik, V., \& Wingreen, N. S. (2012). Responding to chemical gradients: bacterial chemotaxis. Current Opinion in Cell Biology, 24(2), 262-268. doi:doi.org/10.1016/j.ceb.2011.11.008 
Sutherland, E. W., \& Rall, T. W. (1958). Fractionation and Characterization of a Cyclic Adenine Ribonucleotide Formed by Tissue Particles. Journal of Biological Chemistry, 232(2), 10771091.

Typas, A., \& Sourjik, V. (2015). Bacterial protein networks: properties and functions. Nat Rev Microbiol, 13(9), 559-572. doi:10.1038/nrmicro3508

Ulrich, L. E., Koonin, E. V., \& Zhulin, I. B. (2005). One-component systems dominate signal transduction in prokaryotes. Trends in Microbiology, 13(2), 52-56. doi:10.1016/j.tim.2004.12.006

van Duijn, M., Keijzer, F., \& Franken, D. (2006). Principles of minimal cognition: Casting cognition as sensorimotor coordination. Adaptive Behavior, 14(2), 157-170. doi:10.1177/105971230601400207

Västermark, Å., \& Saier, M. H. (2014). The involvement of transport proteins in transcriptional and metabolic regulation. Current Opinion in Microbiology, 18, 8-15. doi:https://doi.org/10.1016/j.mib.2014.01.002

Webre, D. J., Wolanin, P. M., \& Stock, J. B. (2003). Bacterial chemotaxis. Current Biology, 13(2), R47-R49. doi:10.1016/s0960-9822(02)01424-0

Winning, J., \& Bechtel, W. (2018). Rethinking causality in neural mechanisms: Constraints and control. Minds and Machines, 28(2), 287-310.

\section{About the Authors}

Leonardo Bich is a 'Ramon y Cajal' Researcher at the IAS-Research Centre of the University of the Basque Country (Spain). He obtained a PhD in Epistemology of Complex Systems from the University of Bergamo (Italy). He worked at the CNRS (France), at the Biology of Cognition Lab of the Universidad de Chile, and at the Center for Philosophy of Science of the University of Pittsburgh. His research is focused on theoretical and epistemological issues related to biological organisation, autonomy, and control and on their implications for investigations in Origins of Life, Synthetic and Systems Biology, and Theoretical Biology.

William Bechtel is Distinguished Professor in the Department of Philosophy and a member of the Center for Circadian Biology and the Interdisciplinary Program in Cognitive Science at the University of California, San Diego. His research addresses discovery and explanation in the life sciences, including neuroscience and cognitive science. He is co-author of Discovering Complexity: Decomposition and Localization as Strategies in Scientific Research (Princeton, 1993, MIT Press, 2010) and Philosophy of Neuroscience (Cambridge, 2022), and author of Discovering cell mechanisms: The Creation of Modern Cell Biology (Cambridge, 2006) and Mental mechanisms: Philosophical Perspectives on Cognitive Neuroscience (Routledge, 2008). 